CASE REPORT

\title{
Investigating the Characteristics of Covert Unilateral Spatial Neglect Using the Modified Posner Task: A Single-subject Design Study
}

\author{
Shinpei Osaki, RPT, MS a,b Kazu Amimoto, RPT, PhD ${ }^{\text {b }}$ Yasuhiro Miyazaki, RST, PhD a \\ Junpei Tanabe, RPT, MS ${ }^{b}$ and Nao Yoshihiro, ROT, MS ${ }^{b}$
}

\begin{abstract}
Objectives: Patients identified as asymptomatic for unilateral spatial neglect (USN) based on paper-and-pen tests nonetheless often collide with objects to their left while walking. This study aimed to investigate chronic USN in subjects who experienced collisions while walking. Methods: Two patients with chronic USN who experienced collisions while walking were evaluated using the Behavioral Inattention Test-conventional (BIT-c). Additionally, the modified Posner task (MPT) was used to evaluate the left and right reaction times. MPT targets randomly appeared either on the side indicated by the cue (valid condition) or on the opposite side (invalid condition). This study used an alternating treatments single-case design. The valid and invalid conditions of the MPT alternated rapidly and randomly to determine differences in reaction time. Statistical analysis compared left and right reaction times using a one-tailed randomization test to study valid and invalid conditions. Results: The total BIT-c score was in the normal range for both subjects, whereas MPT reaction times were higher on the left side than on the right side for the invalid condition. However, for the valid condition, only Case B had increased reaction times on the left side. Conclusions: The MPT valid condition evaluates voluntary attention, whereas the invalid condition evaluates the reorientation of attention. Consequently, for Case $\mathrm{A}$, a left reorientation of attention deficit was observed, whereas, for Case B, left voluntary attention and left reorientation of attention deficits were observed. The MPT results revealed the characteristics of covert neglect signs. USN evaluation would benefit from additional research using MPT.
\end{abstract}

Key Words: covert neglect; modified Posner task; single-case experimental design study; unilateral spatial neglect

\section{INTRODUCTION}

Unilateral spatial neglect (USN) is defined as the absence of spatial attention and incoming stimuli on the contralateral side to the stroke lesion site. ${ }^{1)}$ USN was observed in $43 \%$ of stroke patients with right hemisphere lesions. ${ }^{2)}$ Spatial attention can be divided into two categories: voluntary attention and reorientation of attention. ${ }^{3-5)}$ Voluntary attention enhances the process of patients' prediction and response, whereas reorientation of attention requires an operation to switch the attention directed from one side to the other. These two types of attention are tested using a reaction-time task; however, in the clinical setting, a simple paper test is usually carried out.

Several paper-and-pencil tests have been employed to evaluate the exact status of USN on the left extremity, including the most routinely used tests, such as the Behavioral Inattention Test-conventional subtest (BIT-c). BIT-c is a paper-and-pencil measurement of USN that includes several subtests (cancellation tasks, figure copying, drawing, and

Received: November 18, 2020, Accepted: February 10, 2021, Published online: March 6, 2021

a Department of Rehabilitation, Kansai Electric Power Hospital, Osaka, Japan

${ }^{\mathrm{b}}$ Graduate School of Human Health Sciences, Tokyo Metropolitan University, Tokyo, Japan

Correspondence: Shinpei Osaki, RPT, MS, Graduate School of Human Health Sciences, Tokyo Metropolitan University, 7-2-10 Higashiogu, Arakawa-ku, Tokyo 116-8551, Japan, E-mail: pt8jfh1151s.o@gmail.com

Copyright (C) 2021 The Japanese Association of Rehabilitation Medicine

This is an open-access article distributed under the terms of the Creative Commons Attribution Non-Commercial No

Derivatives (CC BY-NC-ND) 4.0 License. http://creativecommons.org/licenses/by-nc-nd/4.0/ 

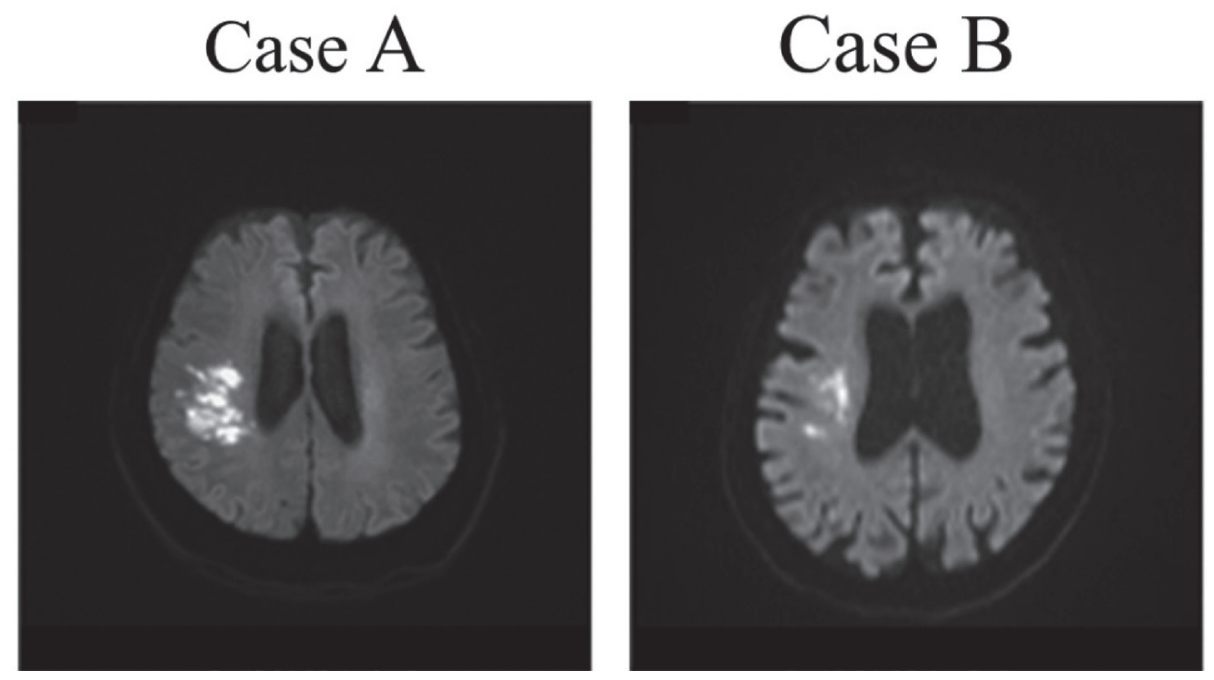

Fig. 1. Brain magnetic resonance imaging of Case A and Case B.

Table 1. Characteristics of the two participants

\begin{tabular}{lcc}
\hline Clinical data & Case A & Case B \\
\hline Age (years) & 84 & 76 \\
Sex & Female & Female \\
Dominant hand & Right & Right \\
Educational history (years) & 12 & 12 \\
Lesion area & Right hemisphere & Right hemisphere \\
\hline
\end{tabular}

line bisection). ${ }^{6}$ High BIT-c scores correspond to a better attentional performance and an absence of neglect symptoms (score range, 0-146). These paper-and-pencil tests have been used as a measure of poor performance in daily activities.7) However, the results of these tests may sometimes not reflect the correct status of neglect behavior in daily activities. ${ }^{8)}$

USN patients often collide with people or objects located on their left side, such as doors and furniture, while walking or driving a wheelchair.9) While walking or driving a wheelchair, the reaction time to people or objects on the left side is important. However, reaction times are not evaluated in paper-and-pencil tests.

Recently, reaction times evaluated using the Posner task in patients with USN have been reported. ${ }^{10,11)}$ The Posner task is a method used to examine spatial attention by measuring reaction times and is used to examine voluntary attention and reorientation of attention. Voluntary attention is required to predict where a person will come out of a door while walking, for example, whereas reorientation of attention is necessary when a person emerges from the opposite direction to that expected while walking. In this situation, the patient needs a quick reaction time for both types of attention. Patients with acute USN showed increased reaction times for tasks associated with spatial attention, even those who were asymptomatic on a paper-and-pencil test. ${ }^{12,13)}$ However, whether walking collisions are associated with reaction times remains unclear. Furthermore, only a few studies have reported increased reaction times that remain in patients with chronic-phase USN. ${ }^{11)}$ Therefore, we investigated reaction-time tasks in two patients with chronic USN who were asymptomatic on paper-and-pencil tests but had collisions while walking nonetheless.

\section{METHODS}

\section{Subjects}

We recruited two right-handed women who had been diagnosed with right cerebral infarction and left hemiplegia. Their characteristics are shown in Fig. 1 and Table 1. Both were inpatients at Kansai Electric Power Hospital and were diagnosed with left USN. The patients were clinically examined and assessed using BIT-c and the Catherine Bergego 
Table 2. Neuropsychological and neglect evaluations

\begin{tabular}{|c|c|c|c|c|}
\hline \multirow{2}{*}{$\begin{array}{l}\text { Clinical data } \\
\text { Time after onset (days) }\end{array}$} & \multicolumn{2}{|c|}{ Case A } & \multicolumn{2}{|c|}{ Case B } \\
\hline & 52 & 181 & 42 & 174 \\
\hline FMA-LE motor function (/34) & 24 & 28 & 22 & 30 \\
\hline sensory function $(/ 12)$ & 12 & 12 & 12 & 12 \\
\hline MAS for the affected lower limbs & 0 & 0 & 0 & 0 \\
\hline Side of spatial neglect & Left & & Left & \\
\hline BIT-c total score $(/ 146)$ & 128 & 143 & 129 & 142 \\
\hline 1. Line crossing & 36 & 36 & 36 & 36 \\
\hline 2. Letter cancellation & 29 & 36 & 36 & 38 \\
\hline 3. Star cancellation & 50 & 54 & 51 & 53 \\
\hline 4. Figure and shape copying & 3 & 4 & 0 & 4 \\
\hline 5. Line bisection & 8 & 9 & 7 & 9 \\
\hline 6. Representation drawing & 2 & 3 & 1 & 3 \\
\hline CBS objective total score $(/ 30)$ & 9 & 2 & 8 & 3 \\
\hline 1. Gaze orientation & 1 & 0 & 0 & 0 \\
\hline 2. Limb awareness & 3 & 0 & 2 & 1 \\
\hline 3. Auditory attention & 1 & 0 & 1 & 0 \\
\hline 4. Personal belongings & 0 & 0 & 0 & 0 \\
\hline 5. Dressing & 1 & 0 & 1 & 0 \\
\hline 6. Grooming & 1 & 0 & 0 & 0 \\
\hline 7. Navigation & 0 & 0 & 1 & 0 \\
\hline 8. Collisions & 2 & 2 & 1 & 1 \\
\hline 9. Meals & 0 & 0 & 0 & 0 \\
\hline 10. Cleaning after meals & 0 & 0 & 2 & 1 \\
\hline CBS subjective total score $(/ 30)$ & 1 & 1 & 0 & 0 \\
\hline CBS.diff (anosognosia score) & 8 & 1 & 8 & 3 \\
\hline
\end{tabular}

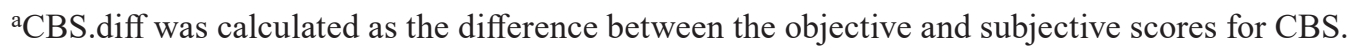

FMA-LE, Fugl-Meyer Assessment Lower Extremity; MAS, Modified Ashworth Scale; BIT-c, Behavioral Inattention Testconventional; CBS, Catherine Bergego Scale.

Scale (CBS), the latter being a more specific measurement for neglect-related functional disability. ${ }^{14)}$ The CBS score is directly based on observations of the patient's function in ten real-life situations, as assessed by physiotherapists, occupational therapists, and nurses. Each of the ten categories in CBS is scored between 0 (no neglect) and 3 (severe neglect); the maximum total score is 30 points. Moreover, CBS measures the anosognosia score by evaluating the difference between the examiner's observations and the patient's selfrating score.

Both patients had mild USN (BIT-c score, 101-130) early after onset but had improved by about 6 months after onset. However, they continued to show USN behavior on assessment. Their cognitive function was within the normal range based on the Mini-Mental State Examination, and no clinically detectable hemianopia was observed by confrontation visual field examination. The purpose of the current study was explained to the subjects, and written consent was obtained in accordance with the Helsinki Declaration. The study was approved by the ethics committee of Kansai Electric Power Hospital (approval number: 19-088).

\section{Case A}

Case A involved an 84-year-old woman with cerebral infarction. A magnetic resonance imaging (MRI) examination revealed lesions in the parietal lobes of the right hemisphere. On day 52 after onset, the patient's basic movements required assistance by another person and she walked using a T-handle cane. The patient collided with people and objects located on her left side while walking. This patient scored $128 / 146$ on the BIT-c and 9/30 on the CBS, indicating USN (Table 2). On day 180 after onset, she was able to walk using 
a T-handle cane without assistance. However, she still collided with people and objects on her left side while walking.

\section{Case B}

Case B involved a 76-year-old woman with cerebral infarction. MRI examinations showed lesions in the parietal lobes of the right hemisphere. On day 42 after onset, the patient's basic movements required assistance and she could walk with moderate support from another person using a quad cane. The patient collided with people and objects on her left side while walking. This patient scored 129/146 on the BIT-c and $8 / 30$ on the CBS, indicating USN (Table 2). On day 173 after the onset, she was able to walk independently using a quad cane. However, she still collided with people or objects on her left side while walking and disregarded her left field of vision.

\section{Procedure}

In addition to using BIT-c and CBS, the bells cancellation test, the fluff test, and the modified Posner task (MPT) were also performed in Case A (181 days after the onset) and in Case B (174 days after the onset). These additional neglect evaluations were performed because the subjects had no USN symptoms according to BIT-c; however, problems with USN were evident as the subjects performed their activities of daily living (ADLs). The bells cancellation test was selected due to its more intense stimuli than the BIT-c cancellation tasks. MPT is also used to evaluate reaction times, which is not included in BIT-c. The fluff test is a personal neglect assessment tool that particularly evaluates the left side of the body, but it is not included in BIT-c. All evaluations were completed in one day.

\section{Added Neglect Evaluation}

Bells cancellation test. The bells cancellation test is an instrument that uses distractors for the visual exploration of a horizontally oriented A4-sized sheet of paper. ${ }^{15)}$ The test consists of 315 stimuli, with 280 distractors (houses and horses, among others) and 35 target stimuli, which are bell shaped. All icons are black, and the test takes less than $5 \mathrm{~min}$ to complete. The recorded score is the total number of bells circled. The maximum score is 35 . The omission of four or more bells on the right or left half of the page indicates USN. ${ }^{16)}$

Fluff test. This is a test for personal neglect affecting the space on the surface of the patient's body. ${ }^{17)}$ During the test, six targets are attached to each of the blindfolded patient's left arm, leg, and trunk. The total number of targets were 24 identical disks ( $2 \mathrm{~cm}$ in diameter) made of white cardboard. There was Velcro on one side of the disks so that they could be easily attached to clothes. Patients were instructed to remove the targets with their right hand. The omission of two or more Velcro disks on the left half of the patient's body indicates personal neglect.

MPT. The Posner task is a computerized, two-sided reaction-time test assessing the left and right sides. It is a neuropsychological test often used to assess changes in attention. ${ }^{18)}$ The task was modified by extending the target presentation time performed by patients with USN (Fig. 2).

Stimuli were generated using a computer (ASUS ROG STRIX GL703VM) and displayed on a 17.3-inch monitor (refresh rate of $120 \mathrm{~Hz}$ ). Behavioral responses were acquired through a numeric keypad (ELECOM TK-TCM011, Osaka, Japan) interfaced with the computer. Software (Cedrus Corporation SuperLab 5.0, San Pedro, USA) was used to create a modified Posner task. Patients sat at approximately $50 \mathrm{~cm}$ from the monitor. The display contained a central fixed cross (the fixation point) and four square frames orientated to the left and right sides along the horizontal meridian. The diameter of each square was within a $1^{\circ}$ viewing angle, with a circular target at the center. The diameter of each target subtended a visual angle of $0.3^{\circ}$. The central targets in the four peripheral squares were placed at a distance with a visual angle of $4.3^{\circ}$ from the fixation point.

The start of a new trial was indicated by the color change of the fixed circle from red to green. Then, $500 \mathrm{~ms}$ later, an arrow cue pointing to the left or right appeared at the fixation point for $2000 \mathrm{~ms}$. After a delay of 1000-2000 ms, the target (circle) appeared within any of the four frames (left up, left down, right up, or right down) for $3000 \mathrm{~ms}$ or until a response was recorded. The target appeared in the location indicated by the cue in $80 \%$ of the trials (valid condition), whereas it appeared on the opposite side in $20 \%$ of the trials (invalid condition). The two patients were asked to detect the target as quickly as possible and to press the response key with their right hand. The key reaction time and accuracy of the key press timing were recorded. Subsequent trials were performed, separated by a rest interval of $1000 \mathrm{~ms}$. Each block contained 60 trials (48 valid, 12 invalid). Each patient completed two blocks. The test lasted a total of 15 min including practice exercises. Initially, for practice, we explained that the button should be pressed when the target appeared in any of the four squares. After this explanation, the patients performed 12 practice trials and then performed the actual test. 


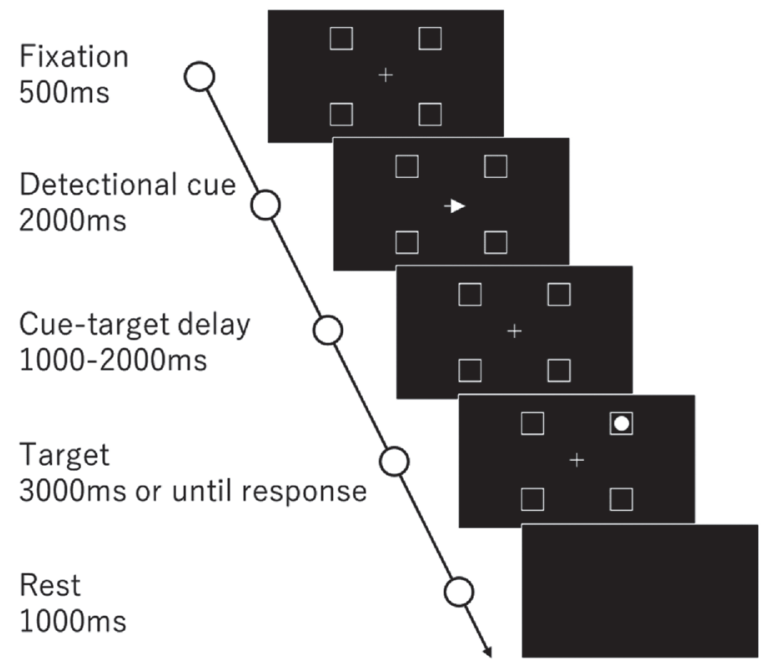

Valid condition

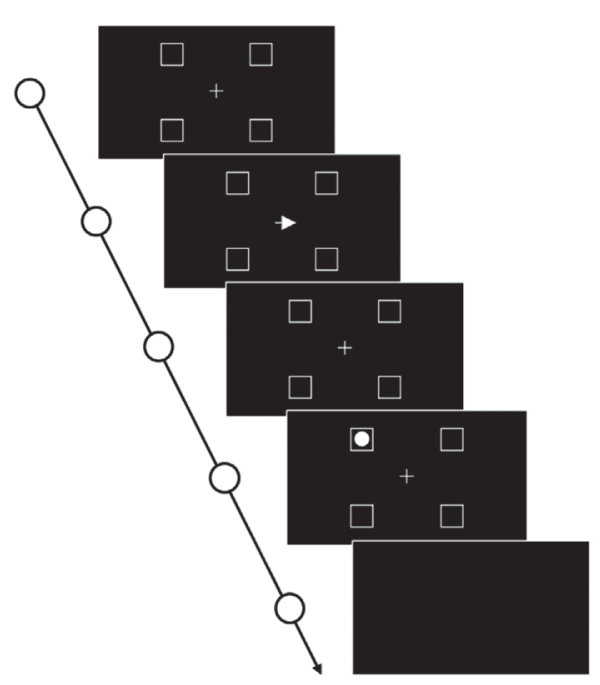

Invalid condition

Fig. 2. Modified Posner task screen used in the present experiment. This task displayed a fixation screen for $500 \mathrm{~ms}$, and then for $2000 \mathrm{~ms}$, an arrow cue pointing to the left or right was displayed. After a delay of 1000-2000 ms, a target (circle) appeared within one of the four frames (left up, left down, right up, or right down) for $3000 \mathrm{~ms}$ or until a response was recorded. The target appeared on the side indicated by the cue in $80 \%$ of trials (valid condition) and on the opposite side in $20 \%$ of trials (invalid condition).

\section{Experimental Design}

This study used an alternating treatments design. The alternating treatment design is a single-case study design consisting of rapid, random or semi-random changes in two or more conditions, each with approximately equal probability of being present during each measurement opportunity. ${ }^{19)}$ Alternating treatment designs can be analyzed by randomization tests. ${ }^{20)}$ The valid and invalid conditions of the MPT alternated rapidly and randomly to determine the difference in reaction time. The MPT resulted in 120 data points from 2 blocks for each patient.

\section{Data Analysis}

The MPT analyzes spatial attention through rates of correct detection and average reaction times for four conditions: (a) the target appeared on the upper left or lower left, and the cue was to the left (position, left; validity, valid); (b) the target appeared on the upper left or lower left, but the cue was to the right (position, left; validity, invalid); (c) the target appeared on the upper right or lower right, and the cue was to the right (position, right; validity, valid); (d) the target appeared on the upper right or lower right, but the cue was to the left (position, right; validity, invalid).

Statistical analysis compared the left and right reaction times using a one-tailed randomization test to study valid and invalid conditions. A randomized test is a procedure to determine the significance by calculating the test statistic $\mathrm{T}$ for one-subject experimental data. ${ }^{21,22)}$ The statistical significance level was set at $\mathrm{P}<0.05$. The software used for the analyses was RT4WIN for Windows. ${ }^{23)}$

\section{RESULTS}

\section{BIT-c and CBS}

About 6 months after stroke onset, Case A scored 143/146 on BIT-c, with all six items indicating no USN; however, on item 8 of CBS (Collision with people or objects on the left side while walking) the score was $2 / 30$, indicating USN (Table 2). Also 6 months after stroke onset, Case B scored $142 / 146$ on BIT-c, with all six items indicating no USN, but scored $3 / 30$ on CBS, which indicated the presence of USN. Case B scored 1 point each for the following CBS items: item 2 (forgets about the left part of her body), item 8 (collision with people or objects on the left side while walking), and item 10 (forgets to clean the left side of her mouth after eating) (Table 2). Both subjects were observed to collide with people or objects on their left side while walking when their attention was turned to the right. Case A did not collide with 
people or objects during walking with voluntary attention. In contrast, Case B experienced collisions even when she was using voluntary attention.

\section{Bells Cancellation Test}

Both patients had no errors in this test, with perfect scores. The test duration was $3 \mathrm{~min}$ and $38 \mathrm{~s}$ for Case A and $3 \mathrm{~min}$ and $24 \mathrm{~s}$ for Case B.

\section{Fluff Test}

For Case A, only one target on the left half of the body was omitted, which did not indicate personal neglect; the undetached target was on the upper left arm. In contrast, for Case B, three targets on the left half of the body were omitted, which indicated personal neglect; the undetached targets were two on the upper left arm and one on the trunk.

\section{MPT}

The detection rates for Cases A and B were 100\% for all four conditions; Figure 3 shows the reaction-time results for each trial of the two patients individually for the four different target-cue conditions. Figure 4 shows the average reaction times for each patient individually for the four different target-cue conditions. For the average reaction time, a randomization test revealed the main effects of left-right differences in the valid and invalid conditions. For Case A, no statistical difference was observed between the left and right sides for valid conditions (left vs. right: $530 \pm 95$ vs. $503 \pm 67$, $\mathrm{t}=1.59$, n.s.). However, for the invalid condition, the reaction time was significantly higher for the left side than for the right (left vs. right: $640 \pm 87$ vs. $522 \pm 42, \mathrm{t}=4.04, \mathrm{P}<0.01$ ). For Case B, the reaction time for the valid condition was significantly higher on the left side than on the right (left vs. right: $556 \pm 186$ vs. $492 \pm 94, \mathrm{t}=2.14, \mathrm{P}<0.01$ ). Furthermore, the reaction time for the invalid condition was significantly higher on the left side than on the right (left vs. right: $646 \pm$ 105 vs. $535 \pm 49, \mathrm{t}=3.40, \mathrm{P}<0.01)$.

\section{DISCUSSION}

The two patients were found to be subclinical according to the total BIT-c score, and all subitems were above the cutoff values for USN; however, problematic neglect behavior was still observed. Additional neglect evaluations were carried out and neglect behavior was detected.

First, interpretation of the additional evaluation results of the two patients is given. The total scores of the bells cancellation test, which is more sensitive than paper-and- pencil tests, exceeded the cutoff value for USN in both cases. Directional attention is strongly associated with cancellation tests. ${ }^{24)}$ In both of our subjects, the bells cancellation test showed no left directional attention impairment. MPT is a method used to evaluate directional attention based on reaction times. For both subjects, the reaction time on the left was higher than that on the right, which is consistent with the findings in previous reports. ${ }^{11,13)}$ However, for the valid condition, only Case B had an increased reaction time on the left side. The valid condition assesses voluntary attention, whereas the invalid condition assesses the reorientation of attention. ${ }^{3,25)}$ Thus, in Case A, a left reorientation of attention deficit was observed, whereas in Case B, left voluntary attention and left reorientation of attention deficits were observed. Furthermore, no directional attention deficit was found in the bells cancellation test, although such a deficit was detected using MPT. The fluff test results did not indicate a problem in Case A, but in Case B, three targets on the left side were missed, indicating personal neglect. Personal neglect is the closest neglect behavior to the body. ${ }^{26)}$ BIT-c does not assess personal neglect; consequently, its occurrence was not confirmed.

Second, the CBS problem is interpreted based on the results of the additional evaluations. According to gait observations in the two subjects, collisions with people or objects on the left side occurred when attention was directed to the right side. This is the result of a reorientation of attention deficit. Findings for the invalid condition in MPT, which are also shown in the above interpretation, support the presence of a reorientation of attention deficit in both subjects. Therefore, reorientation of attention deficits result in collisions while walking. However, only Case B had a left-sided voluntary attention deficit. Voluntary attention is required as a USN compensation strategy in which patients focus their attention to the left side to avoid collisions while walking. ${ }^{27-29)}$ In Case $\mathrm{B}$, this compensation strategy to avoid collisions while walking could not be performed due to their voluntary attention deficit. Furthermore, only Case B had personal neglect, a left body cognitive defect associated with left collision while walking.

Finally, the difference in MPT results between the two patients with brain lesions was interpreted. Primary lesions in the superior longitudinal fasciculus (SLF) are known to be involved in USN. ${ }^{30)}$ Imaging findings showed damage to the SLF in both cases (Fig. 1). The SLF is associated with both voluntary attention and the reorientation of attention. ${ }^{31)}$ However, the laterality of voluntary attention reportedly improves in the chronic phase. ${ }^{11)}$ In other words, in both subjects, the 

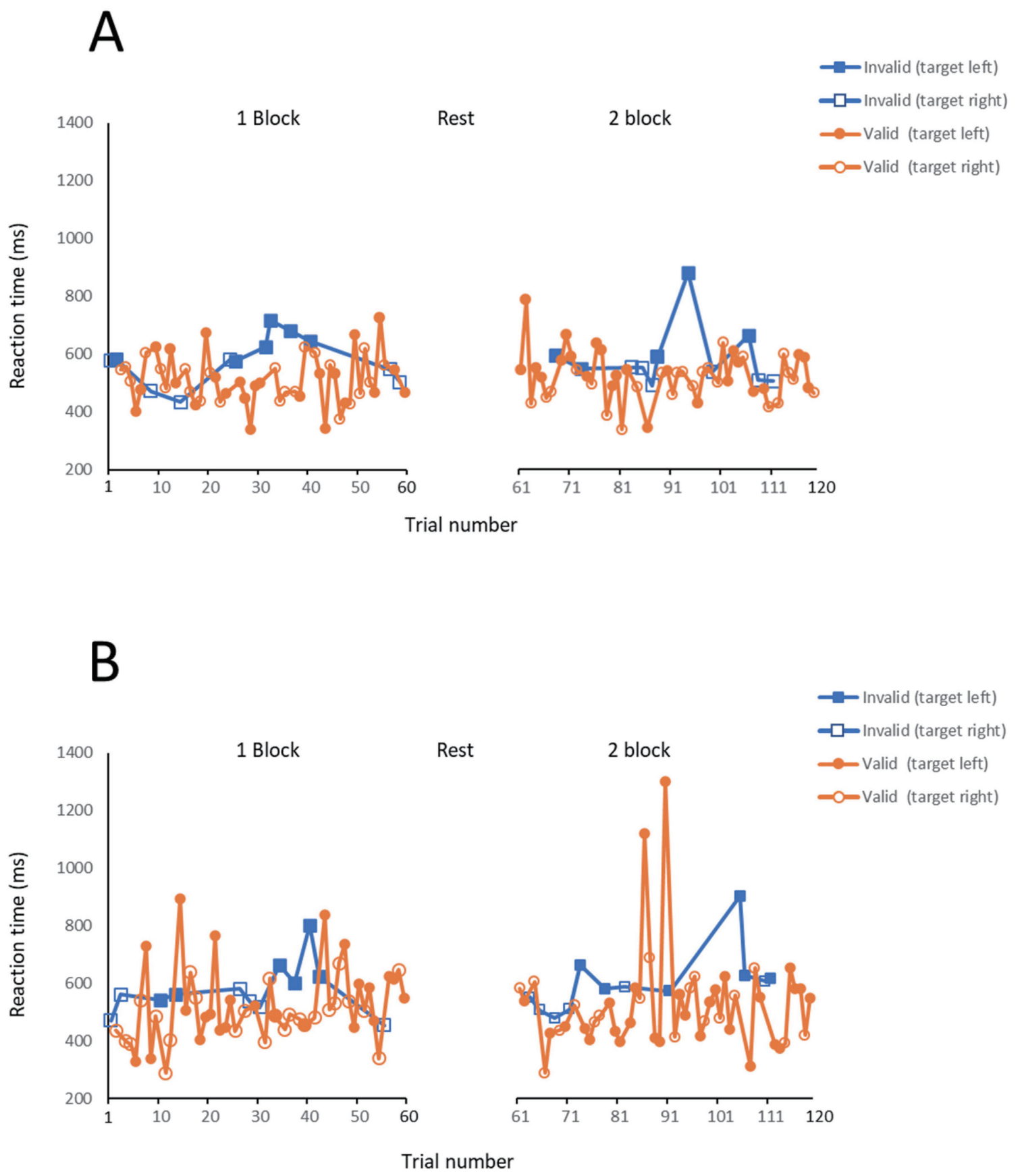

Fig. 3. Modified Posner task reaction time results for each trial. (A) The results of a total of 120 trials in two blocks for Case A. (B) The results of a total of 120 trials in two blocks for Case B.

reaction-time delay improved for the valid condition, which evaluates voluntary attention, whereas the reaction time on the left side was delayed only for the invalid condition, which evaluates the reorientation of attention. However, this result was considered to reflect the difference between the anosognosia scores of the two subjects, i.e., only Case B had a delay on the left side for the valid condition, unlike the findings in a previous study. ${ }^{11)}$ Anosognosia scores were more severe in Case B than in Case A (Table 2). Anosognosia is believed to affect voluntary attention because it implies difficulty in recognizing one's condition and focusing attention on the left side. In Case B, there was almost no awareness of collisions on the left side in daily activities. In contrast, Case A had a mild anosognosia score, and it may have been possible 
A

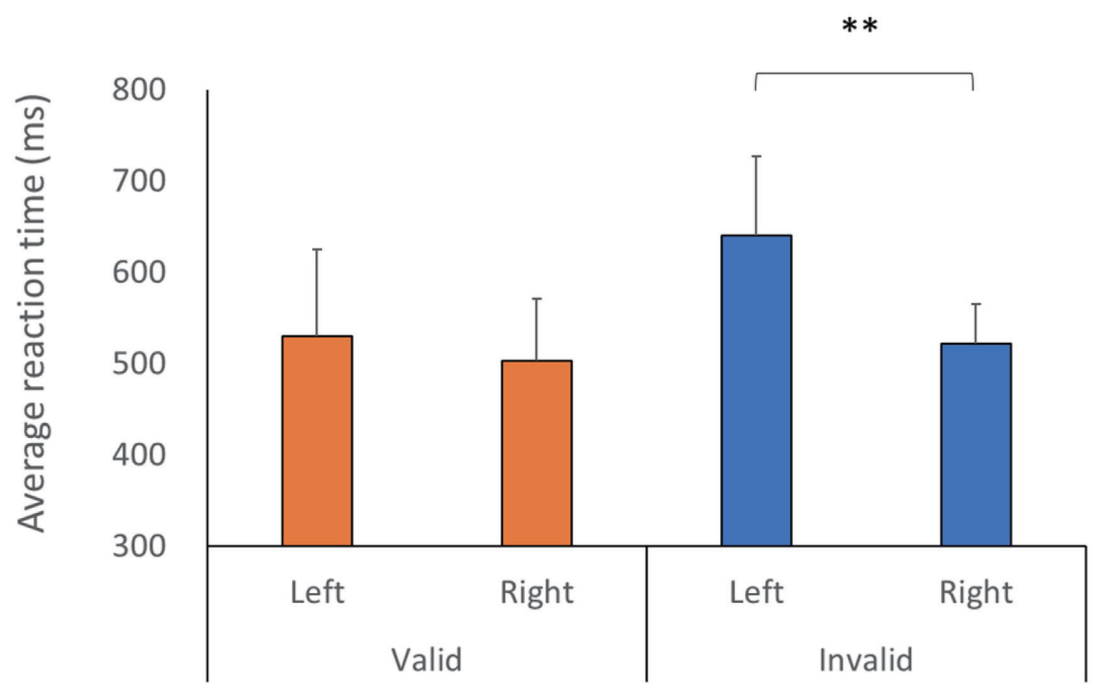

B

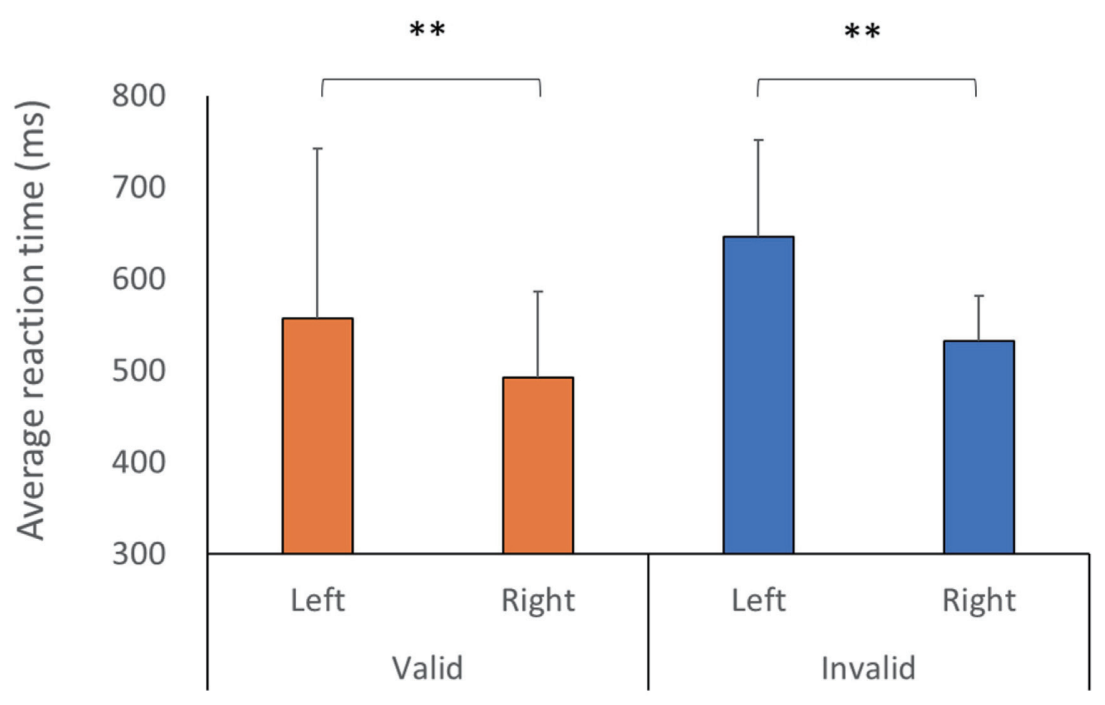

Fig. 4. Average reaction times for the modified Posner task. Comparison of the left and right reaction times for Case A (A) and Case B (B) for the modified Posner task for valid and invalid conditions. Bars show mean values and standard deviations. **Statistically significant $(\mathrm{P}<0.01)$.

for her to voluntarily focus attention to the left side. Case B was younger than Case A but had cerebral atrophy (Fig. 1). Anosognosia occurs from a wide range of affected brain regions, such as the SLF, the frontal lobe, the temporoparietal junction, and the insular cortex. ${ }^{32)}$ The symptoms of anosognosia in Case B may have resulted from widespread functional decline caused by cerebral atrophy in addition to cerebral infarction in the SLF region. 


\section{LIMITATIONS}

A major limitation of this report is that only two subjects were considered; therefore, additional cases should be investigated in the future. Furthermore, there may be reliability problems with USN tests other than the ones evaluated here. Moreover, in this report, hemianopia was diagnosed using a confrontation visual field examination; a detailed evaluation using Goldman's perimeter would be beneficial. Therefore, further investigations into USN in a larger number of subjects are needed.

\section{CONCLUSIONS}

In summary, reaction-time tasks were carried out in two subclinical patients who did not have USN according to the results of BIT-c but who displayed neglect behavior. USN evident during ADLs can require additional evaluation using MPT and the fluff test.

\section{ACKNOWLEDGMENTS}

This project was made possible thanks to the cooperation of the participants.

\section{CONFLICTS OF INTEREST}

The authors declare that there are no conflicts of interest.

\section{REFERENCES}

1. Mesulam MM: A cortical network for directed attention and unilateral neglect. Ann Neurol 1981;10:309-325. DOI:10.1002/ana.410100402, PMID:7032417

2. Corbetta M, Kincade MJ, Lewis C, Snyder AZ, Sapir A: Neural basis and recovery of spatial attention deficits in spatial neglect. Nat Neurosci 2005;8:1603-1610. DOI:10.1038/nn1574, PMID:16234807

3. Corbetta M, Kincade JM, Ollinger JM, McAvoy MP, Shulman GL: Voluntary orienting is dissociated from target detection in human posterior parietal cortex. Nat Neurosci 2000;3:292-297. DOI:10.1038/73009, PMID:10700263

4. Macaluso E, Doricchi F: Attention and predictions: control of spatial attention beyond the endogenousexogenous dichotomy. Front Hum Neurosci 2013;7:685. DOI:10.3389/fnhum.2013.00685, PMID:24155707
5. Corbetta M, Shulman GL: Spatial neglect and attention networks. Annu Rev Neurosci 2011;34:569-599. DOI:10.1146/annurev-neuro-061010-113731, PMID:21692662

6. Wilson B, Cockburn J, Halligan P: Development of a behavioral test of visuospatial neglect. Arch Phys Med Rehabil 1987;68:98-102. PMID:3813864

7. Cherney LR, Halper AS, Kwasnica CM, Harvey RL, Zhang M: Recovery of functional status after right hemisphere stroke: relationship with unilateral neglect. Arch Phys Med Rehabil 2001;82:322-328. DOI:10.1053/ apmr.2001.21511, PMID:11245753

8. Seron X, Deloche G, Coyette F: A retrospective analysis of a single case neglect therapy: A point of theory. In Seron X, Deloche G (Eds.), Neuropsychology and Neurolinguistics. Cognitive Approaches in Neuropsychological Rehabilitation. 1989:289-316.

9. Azouvi P: Functional consequences and awareness of unilateral neglect: study of an evaluation scale. Neuropsychol Rehabil 1996;6:133-150. DOI:10.1080/713755501

10. Deouell L, Sacher Y, Soroker N: Assessment of spatial attention after brain damage with a dynamic reaction time test. J Int Neuropsychol Soc 2005;11:697-707. DOI:10.1017/S1355617705050824, PMID:16248905

11. Rengachary J, d'Avossa G, Sapir A, Shulman GL, Corbetta M: Is the Posner reaction time test more accurate than clinical tests in detecting left neglect in acute and chronic stroke? Arch Phys Med Rehabil 2009;90:2081-2088. DOI:10.1016/j.apmr.2009.07.014, PMID:19969172

12. Bonato $\mathrm{M}$, Priftis $\mathrm{K}$, Umiltà $\mathrm{C}$, Zorzi M: Computerbased attention-demanding testing unveils severe neglect in apparently intact patients. Behav Neurol 2013;26:179-181. DOI:10.1155/2013/139812, PMID:22713418

13. Machner B, Könemund I, von der Gablentz J, Bays PM, Sprenger A: The ipsilesional attention bias in righthemisphere stroke patients as revealed by a realistic visual search task: neuroanatomical correlates and functional relevance. Neuropsychology 2018;32:850865. DOI:10.1037/neu0000493, PMID:30321035

14. Azouvi P, Olivier S, de Montety G, Samuel C, Louis-Dreyfus A, Tesio L: Behavioral assessment of unilateral neglect: study of the psychometric properties of the Catherine Bergego scale. Arch Phys Med Rehabil 2003;84:51-57. DOI:10.1053/apmr.2003.50062, PMID:12589620 
15. Gauthier L, Dehaut F, Joanette Y: The bells cancellation test: a quantitative and qualitative test for visual neglect. Int J Clin Neuropsychol 1989;11:49-54.

16. Azouvi P, Bartolomeo P, Beis JM, Perennou D, PradatDiehl P, Rousseaux M: A battery of tests for the quantitative assessment of unilateral neglect. Restor Neurol Neurosci 2006;24:273-285. PMID:17119304

17. Cocchini G, Beschin N, Jehkonen M: The fluff test: a simple task to assess body representation neglect. Neuropsychol Rehabil 2001;11:17-31. DOI:10.1080/09602010042000132

18. Posner MI: Orienting of attention. Q J Exp Psychol 1980;32:3-25. DOI:10.1080/00335558008248231, PMID:7367577

19. Barlow DH, Hayes SC: Alternating treatments design: one strategy for comparing the effects of two treatments in a single subject. J Appl Behav Anal 1979;12:199-210. DOI:10.1901/jaba.1979.12-199, PMID:489478

20. Onghena P, Edgington ES: Randomization tests for restricted alternating treatments designs. Behav Res Ther 1994;32:783-786. DOI:10.1016/0005-7967(94)90036-1, PMID:7980365

21. Edgington ES: Statistical inference and nonrandom samples. Psychol Bull 1966;66:485-487. DOI:10.1037/ h0023916, PMID:5974621

22. Hunter MA, May RB: Statistical testing and null distributions: what to do when samples are not random. Can J Exp Psychol 2003;57:176-188. DOI:10.1037/ h0087424, PMID:14596476

23. Huo M, Onghena P: RT4Win: A Windows-based program for randomization tests. Psychol Belg 2012;52:387-406. DOI:10.5334/pb-52-4-387

24. Gainotti G, De Luca L, Figliozzi F, Doricchi F: The influence of distracters, stimulus duration and hemianopia on first saccade in patients with unilateral neglect. Cortex 2009;45:506-516. DOI:10.1016/j.cortex.2007.12.008, PMID:19231479
25. Esterman M, Prinzmetal W, DeGutis J, Landau A, Hazeltine E, Verstynen T, Robertson L: Voluntary and involuntary attention affect face discrimination differently. Neuropsychologia 2008;46:1032-1040. DOI:10.1016/j.neuropsychologia.2007.11.014, PMID:18166203

26. Caggiano P, Beschin N, Cocchini G: Personal neglect following unilateral right and left brain damage. Procedia Soc Behav Sci 2014;140:164-167. DOI:10.1016/j. sbspro.2014.04.403

27. Bartolomeo P: The novelty effect in recovered hemineglect. Cortex 1997;33:323-333. DOI:10.1016/S00109452(08)70008-X, PMID:9220262

28. Pflugshaupt T, Bopp SA, Heinemann D, Mosimann UP, von Wartburg R, Nyffeler T, Hess CW, Müri RM: Residual oculomotor and exploratory deficits in patients with recovered hemineglect. Neuropsychologia 2004;42:1203-1211. DOI:10.1016/j.neuropsychologia.2004.02.002, PMID:15178172

29. Hasegawa C, Hirono N, Yamadori A: Discrepancy in unilateral spatial neglect between daily living and neuropsychological test situations: a single case study. Neurocase 2011;17:518-526. DOI:10.1080/13554794.20 10.547506, PMID:21707261

30. Lunven M, Thiebaut De Schotten M, Bourlon C, Duret C, Migliaccio R, Rode G, Bartolomeo P: White matter lesional predictors of chronic visual neglect: a longitudinal study. Brain 2015;138:746-760. DOI:10.1093/ brain/awu389, PMID:25609686

31. Vossel S, Geng JJ, Fink GR: Dorsal and ventral attention systems: distinct neural circuits but collaborative roles. Neuroscientist 2014;20:150-159. DOI:10.1177/1073858413494269, PMID:23835449

32. Monai E, Bernocchi F, Bisio M, Bisogno AL, Salvalaggio A, Corbetta M: Multiple Network Disconnection in Anosognosia for Hemiplegia. Front Syst Neurosci 2020. 29;14:21. 